EPJ Web of Conferences 116, 05004 (2016)

DOI: $10.1051 /$ epjconf/201611605004

(C) Owned by the authors, published by EDP Sciences, 2016

\title{
The data acquisition system for Baikal-GVD
}

A.D. Avrorin ${ }^{1}$, A.V. Avrorin ${ }^{1}$, V.M. Aynutdinov ${ }^{1}$, R. Bannash ${ }^{7}$, I.A. Belolaptikov², D. Yu. Bogorodsky ${ }^{3}$, V.B. Brudanin ${ }^{2}$, N.M. Budnev ${ }^{3}$, I.A. Danilchenko ${ }^{1}$, G.V. Domogatsky ${ }^{1}$, A.A. Doroshenko ${ }^{1}$, A.N. Dyachok ${ }^{3}$, Zh.-A.M. Dzhilkibaev ${ }^{1}$, S.V. Fialkovsky ${ }^{5}$, A.R. Gafarov ${ }^{3}$, O.N. Gaponenko ${ }^{1}$, K.V. Golubkov ${ }^{1}$, T.I. Gress ${ }^{3}$, Z. Honz ${ }^{2}$, K.G. Kebkal ${ }^{7}$, O.G. Kebkal ${ }^{7}$, K.V. Konischev², A.V. Korobchenko ${ }^{2}$, A.P. Koshechkin ${ }^{1}$, F.K. Koshel ${ }^{1}$, A.V. Kozhin ${ }^{4}$, V.F. Kulepov ${ }^{5}$, D.A. Kuleshov ${ }^{1}$, V.I. Ljashuk ${ }^{1}$, M.B. Milenin ${ }^{5}$, R.A. Mirgazov ${ }^{3}$, E.R. Osipova ${ }^{4}$, A.I. Panfilov ${ }^{1}$, L.V. Pan'kov ${ }^{3}$, E.N. Pliskovsky ${ }^{2}$, M.I. Rozanov ${ }^{6}$, E.V. Rjabov ${ }^{3}$, B.A. Shaybonov ${ }^{2}$, A.A. Sheifler ${ }^{1}$, M.D. Shelepov ${ }^{1}$, A.V. Skurihin ${ }^{4}$, A.A. Smagina ${ }^{2}$, O.V. Suvorova ${ }^{1}$, V.A. Tabolenko ${ }^{3}$, B.A. Tarashansky ${ }^{3}$, S.A. Yakovlev ${ }^{7}$, A.V. Zagorodnikov ${ }^{3}$, and V.L. Zurbanov ${ }^{3}$

${ }^{1}$ Institute for Nuclear Research, 60th October Anniversary pr. 7A, Moscow 117312, Russia

2 Joint Institute for Nuclear Research, Dubna 141980, Russia

${ }^{3}$ Irkutsk State University, Irkutsk 664003, Russia

${ }^{4}$ Skobeltsyn Institute of Nuclear Physics MSU, Moscow 119991, Russia

${ }^{5}$ Nizhni Novgorod State Technical University, Nizhni Novgorod 603950, Russia

${ }^{6}$ St. Petersburg State Marine University, St. Petersburg 190008, Russia

${ }^{7}$ EvoLogics GmbH, Berlin, Germany

\begin{abstract}
Baikal-GVD will be a neutrino telescope at the cubic-kilometer scale in Lake Baikal. The first out of 10-12 clusters of the first phase of GVD has been deployed and commissioned in April 2015. This paper describes design and implementation of the dataacquisition system of GVD.
\end{abstract}

\section{Introduction}

Baikal-GVD is a neutrino telescope of a new generation with an effective volume of $\sim 1 \mathrm{~km}^{3}$ [1]. It will consist of functionally independent clusters, each consisting of 8 strings each carrying 36 optical modules (OMs). The OMs consist of pressure-tight glass spheres housing 10" Hamamatsu R7081-100 photomultiplier tubes (PMTs) and the control electronics [2].

The OMs on a string are grouped into sections - the lowest-level DAQ units, each of them serving 12 OMs. A section is a complete detection unit that forms a section trigger and provides data transmission from the OMs to the cluster DAQ center. Each string includes several sections, at present 2, in the future up to 4 . The optimum number and configuration of the sections depend on the distance between the OMs and the instrumented length of the strings. Structuring into sections and clusters provides flexibility in adapting to the actual scientific requirements [3].

This is an Open Access article distributed under the terms of the Creative Commons Attribution License 4.0, which permits unrestricted use, distribution, and reproduction in any medium, provided the original work is properly cited. 


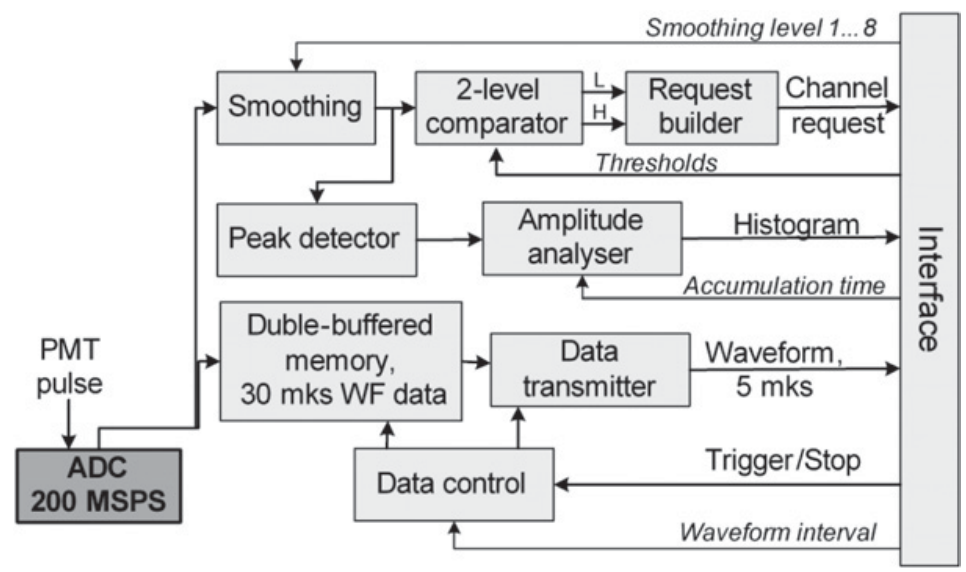

Figure 1. Functional diagram of one measuring channel of the ADC board.

\section{Detection system and digitizing}

The OMs house the PMT and electronics: a high-voltage power supply (SHV 12-2.0 K 1000 P, produced by TRACO Electronic AG), an OM controller (on the basis of a C8051F121 SiLabs micro-controller, designed and manufactured by SNIIP-AUNIS Ltd), a passive PMT divider (18 M $\Omega$ ), a fast two-channel preamplifier and two calibration LEDs (about $470 \mathrm{~nm}$ wavelength). The analog signals from the PMTs pass through a two-channel amplifier and a dedicated deep-water cable $90 \mathrm{~m}$ long and then arrive at the center of the section, at the analog-to-digital converter (ADC) board for digitization and processing.

The analog signals are digitized in 12-channel ADC boards located at the center of the section. The functional diagram of one measuring channel of the ADC board is shown in Fig. 1. Each spectrometric channel is based on an AD9430 ADC with a resolution of 12 bit and a sampling frequency of $200 \mathrm{MHz}$. When digitized, the signal from the ADC is translated into the Xilinx Spartan-6 field-programmable gate array (FPGA). The FPGA software loaded over the data transmission channel ensures control and frontend processing of data arriving from the ADC. The 12 Kbyte FPGA memory buffer allows acquisition of data on the input signal waveform over a time frame as long as $5 \mu \mathrm{s}$.

A peak detector and a multichannel analyzer are connected to the measuring channel. They accumulate monitoring data (amplitude histograms) allowing control of the measuring channel performance. The trigger is based on the information formed in the channels of requests of ADC board. Each channel of requests includes a signal smoothing device (smoothing level, 1-8) and a two-level digital comparator with adjustable thresholds (low threshold L and high threshold $\mathrm{H}$ ). Depending on the stated physical task, the values of the comparator thresholds may vary for different channels and, in normal operating mode of the detector, are 0.3 and 3.0 p.e. for $\mathrm{L}$ and $\mathrm{H}$, respectively. The expected counting rate of an 8 -string cluster at these thresholds is $\sim 100 \mathrm{~Hz}$.

\section{Triggering and data transmission}

An effective suppression of noise events is achieved by using flexible andtunable trigger conditions in the requests analyzer. A Spartan-6 FPGA logic has been configured for generating a trigger, reading data from the ADC channels, and transmitting them over the Ethernet channel (the integrated network 100Base-TX interface) into the data acquisition center.

As a result of processing of $\mathrm{L}$ and $\mathrm{H}$ channel requests from all ADC channels of the section, the requests analyzer forms a request of the section. The embedded hardware-software Coincidence Matrix 


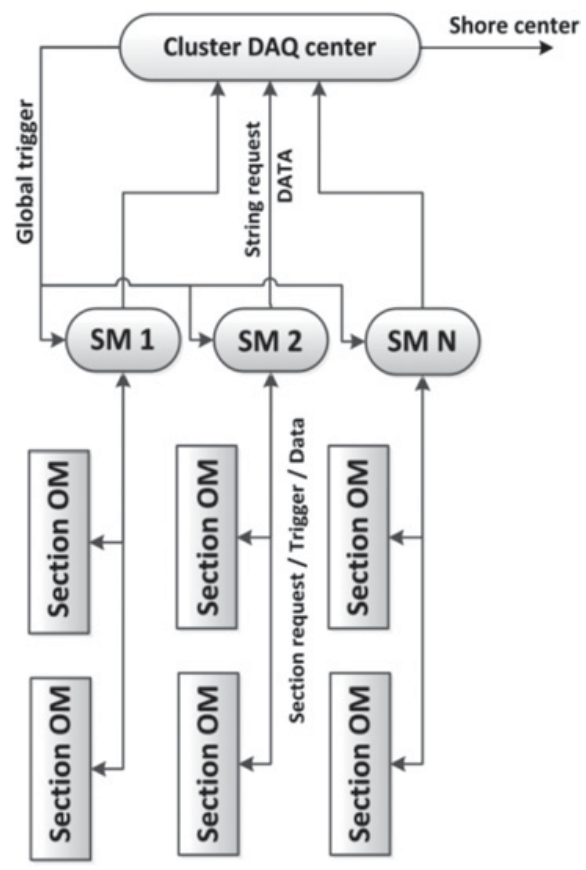

Figure 2. Diagram of the GVD cluster triggering and data transmission.

module $(12 \mathrm{H} \times 12 \mathrm{~L})$ is used to process requests of the channels. This module provides the simplest and fastest method for generating the section request. The trigger system is operative in two modes: majority coincidences of the $\mathrm{L}$ request signals in the selected time interval ("L trigger") and coincidences of the $\mathrm{L}$ and $\mathrm{H}$ requests in any neighboring optical modules (“L\&H trigger”).

The request signals of all sections are sent to the cluster center, where the acknowledgement signal being a common trigger for all strings is generated. The trigger signal initiates the generation of a common signal "Stop" for all measuring channels, read-out of the ADC data buffer, formation of the output buffer (the time frame) and its transmission to the DAQ center. The delay time of the common trigger is $\sim 15 \mu$ s (the delay of the signal passage through 1.2-km line from the central module to the cluster center and backward, as well as the delay in the electronic units). For each channel, the beginning of the time frame may vary within a range of $0-30 \mu$ s relative to the moment of trigger arrival. The Spartan-6 FPGA logic also provides a real time data processing. As a result of this processing, only those parts of the time frame are transmitted to the data acquisition center where an excess over the threshold is observed. In this case, the data compression ratio is as large as $10^{2}$.

The underwater local-area network (LAN) of the Baikal-GVD is a branched structure of star topology, consisting of a group of communication channels for data exchange between different detector units. Each ADC board with a Spartan-6 FPGA chip at the section center has its own IP address in the cluster LAN, which allows access to it and data readout from the registers according to the TCP/IP protocol. The functional diagram of the Baikal-GVD cluster triggering and data transmission system is shown in Fig. 2.

\section{The first Baikal-GVD cluster}

In April 2015 the first cluster of Baikal-GVD was deployed in Lake Baikal and commissioned [1]. The DAQ system of this first full-scale cluster is identical to the data acquisition system of the Gigaton 


\section{EPJ Web of Conferences}

Volume Detector and comprises all assemblies of the future detector. During 2015 the DAQ system worked without critical failures. Some problems with string request transmission will be solved during the next deployment season in 2016.

This work was supported by the Russian Found for Basic Research (grants 13-02-12221, 14-02-00175, 14-0200972).

\section{References}

[1] See the talk of Zhan Dzhilkibaev at this conference

[2] See the talk of Alexey Sheifler at this conference

[3] See the talk of Igor Belolaptikov at this conference 